\title{
The metopic index: an anthropometric index for the quantitative assessment of trigonocephaly from metopic synostosis
}

\author{
Joanna Y. Wang, BA, ${ }^{1}$ Amir H. Dorafshar, MBChB, ${ }^{2}$ Ann Liu, BS, ${ }^{1}$ Mari L. Groves, MD, ${ }^{1}$ and \\ Edward S. Ahn, MD'1
}

1Division of Pediatric Neurosurgery, Department of Neurosurgery, and 2Department of Plastic and Reconstructive Surgery, Johns Hopkins University School of Medicine, Baltimore, Maryland

\begin{abstract}
OBJECTIVE Because the metopic suture normally fuses during infancy, there are varying degrees of severity in head shape abnormalities associated with premature fusion. A method for the objective and reproducible assessment of metopic synostosis is needed to guide management, as current methods are limited by their reliance on aesthetic markers. The object of this study was to describe the metopic index (MI), a simple anthropometric cranial measurement. The measurements can be obtained from CT scans and, more importantly, from palpable cranial landmarks, and the index provides a rapid tool for evaluating patients in both pre- and postoperative settings.
\end{abstract}

METHODS High-resolution head CT scans obtained in 69 patients (age range 0-24 months) diagnosed with metopic craniosynostosis were retrospectively reviewed. Preoperative 3D reconstructions were available in 15 cases, and these were compared with 3D reconstructions of 324 CT scans obtained in a control group of 316 infants (age range 0-24 months) who did not have any condition that might affect head size or shape and also in a subset of this group, comprising 112 patients precisely matched to the craniosynostosis patients with respect to age and sex. Postoperative scans were available and reviewed in 9 of the craniosynostosis patients at a mean time of 7.1 months after surgical repair. 3D reconstructions of these scans were matched with controls based upon age and sex.

RESULTS The mean preoperative MI for patients with trigonocephaly was 0.48 (SD 0.05), significantly lower than the mean values of 0.57 (SD 0.04) calculated on the basis of all 324 scans obtained in controls $(p<0.001)$ and 0.58 (SD 0.04$)$ for the subset of 112 age- and sex-matched controls $(p<0.001)$. For 7 patients with both pre- and postoperative CT scans available for evaluation, the mean postoperative MI was 0.55 (SD 0.03), significantly greater than their preoperative Mls (mean 0.48 [SD 0.04], $p=0.001)$ and comparable to the mean Ml of the controls $(p=0.30)$. In 4 patients, clinically obtained postoperative Mls by caliper measurement were comparable to measurements derived from CT $(p=0.141)$.

CONCLUSIONS The MI is a useful measurement of the severity of trigonocephaly in patients with metopic synostosis. This simple quantitative assessment can potentially be used in the clinical setting to guide preoperative evaluation, surgical repair, and postoperative degree of correction.

http://thejns.org/doi/abs/10.3171/2016.2.PEDS15524

KEY WORDS anthropometric indices; craniosynostosis; metopic synostosis; metopic index; trigonocephaly; craniofacial

$\mathrm{M}$ ETOPIC synostosis is the second most common type of craniosynostosis, and it can be nonsyndromic, occurring in isolation or with fusion of other sutures, or it may occur as part of a dysmorphogenic syndrome such as Crouzon or Saethre-Chotzen syndrome. ${ }^{12,15}$ The metopic suture is the first to fuse physiologically, with complete closure typically occurring by
9 to 11 months. ${ }^{16,17}$ Premature fusion is characterized by restricted growth of the frontal bones, resulting in a prominent midline ridge with a triangular forehead and bitemporal narrowing and occipitoparietal widening-the condition described as trigonocephaly. Compensatory changes in the remaining calvaria result in a characteristic orbital dysmorphology, with depression of the superolateral or-

ABBREVIATIONS EUD = eurion-eurion diameter; FPI = frontoparietal index; MFZ = midfrontozygomatic; $M F Z D=M F Z$ diameter; $M I=$ metopic index. SUBMITTED August 26, 2015. ACCEPTED February 29, 2016.

INCLUDE WHEN CITING Published online May 6, 2016; DOI: 10.3171/2016.2.PEDS15524. 
bital rims and ethmoidal hypoplasia, which manifests as orbital hypotelorism, epicanthal folds, and elevation of the lateral canthal angles. ${ }^{4,15}$

Surgical repair of trigonocephaly, with the goals of restoring anterior cranial fossa volume and improving cosmesis, has evolved considerably over the past few decades, and is typically a collaborative effort between pediatric neurosurgeons and plastic craniofacial surgeons. ${ }^{13}$ However, up to this point, the assessment of metopic synostosis has been largely subjective and involves the use of aesthetic markers, which limits preoperative evaluation of trigonocephaly severity and postoperative assessment of correction. Indeed, one of the most widely used tools to assess craniosynostosis, the Whitaker classification, relies on the judgment of the patient, family, and surgeon to determine the need for surgical correction. ${ }^{19}$ Without an objective grading system, clinical determination of trigonocephaly that requires surgical intervention is challenging. ${ }^{8}$

The use of anthropometric cranial measurements has improved the precision and reliability of the assessment of other cranial deformities such as positional plagiocephaly, scaphocephaly, and brachycephaly. ${ }^{21,22}$ The objective of this study was to describe the metopic index (MI), a simple anthropometric cranial measurement that can be obtained both from CT scans and from palpable cranial landmarks, as a rapid tool for evaluating patients with metopic synostosis in both pre- and postoperative settings.

\section{Methods}

Clinical and radiographic information for 69 patients diagnosed with nonsyndromic single-suture metopic synostosis at our institution between 2003 and 2014 was retrospectively reviewed. All research protocols were approved by our Institutional Review Board for Human Research. Patients with other conditions affecting cranial size and shape, including hydrocephalus and intracranial masses, were excluded. Fifteen patients had preoperative CT scans with adequate slices to create high-resolution 3D reconstructions, and 7 of these patients had postoperative CT scans for comparison.

A total of 324 scans of 316 full-term infants without craniosynostosis or other abnormality affecting head cranial size or shape (age range 0 to 24 months) were reviewed; serial imaging studies were included only if the scans were performed at least 1 month apart. Two scans were included for each of 8 patients, and the remainder of the 292 scans were obtained in 292 distinct individuals. These scans were obtained between 2005 and 2012 in the course of clinical evaluations for trauma, headache, seizure, and change in neurological status. Patients who had a history of conditions affecting cranial size or shape, including hydrocephalus, prematurity, intracranial masses, skeletal diseases affecting the calvaria, and growth disorders, were excluded from the control group.

Using UltraVisual Advanced Visualization software (Emageon), high-resolution 3D reconstructions were created using bone windows. Superior projections were used for linear measurements between anatomical landmarks, which were made by means of the geometrical "polygon tool." The metopic index (MI) consists of the midfronto- zygomatic diameter (MFZD) divided by the eurion-eurion diameter (EuD). The MFZD is the minimum width between the midfrontozygomatic (MFZ) points, or the midpoint along the recess above the brow ridge between the anteroinferolateral frontal bone and frontal bone zygomatic process. The EuD was measured as the maximum cranial width (i.e., distance between the lateral extremes of the skull) (Fig. 1). The available high-resolution head CT scans were reviewed and measurements were performed by 2 observers (J.Y.W. and E.S.A.). Agreement between the 2 reviewers was assessed, and the intraclass correlation coefficient was found to be adequate at 0.82 . The average of the measurements of the 2 reviewers were used for further analysis.

In the clinical setting, the corollary of the above measurement was determined with measuring calipers. Caliper points were placed in the recesses of the brow ridge that were visible from the superior vantage point. In infants with metopic craniosynostosis, these recesses were consistently pronounced and medially displaced compared with the position in infants without craniosynostosis, due to the lateral restriction of the forehead. Available measurements from clinical records were used to compare the clinically obtained measurements from those derived from CT scans.

EuDs, MFZDs, and MIs for patients with metopic synostosis were compared with measurements obtained in the complete set of 324 scans obtained in the control group as well as a subset of 112 scans obtained in exact age-matched controls using the unpaired Student t-test. For patients with pre- and postoperative head CT scans,

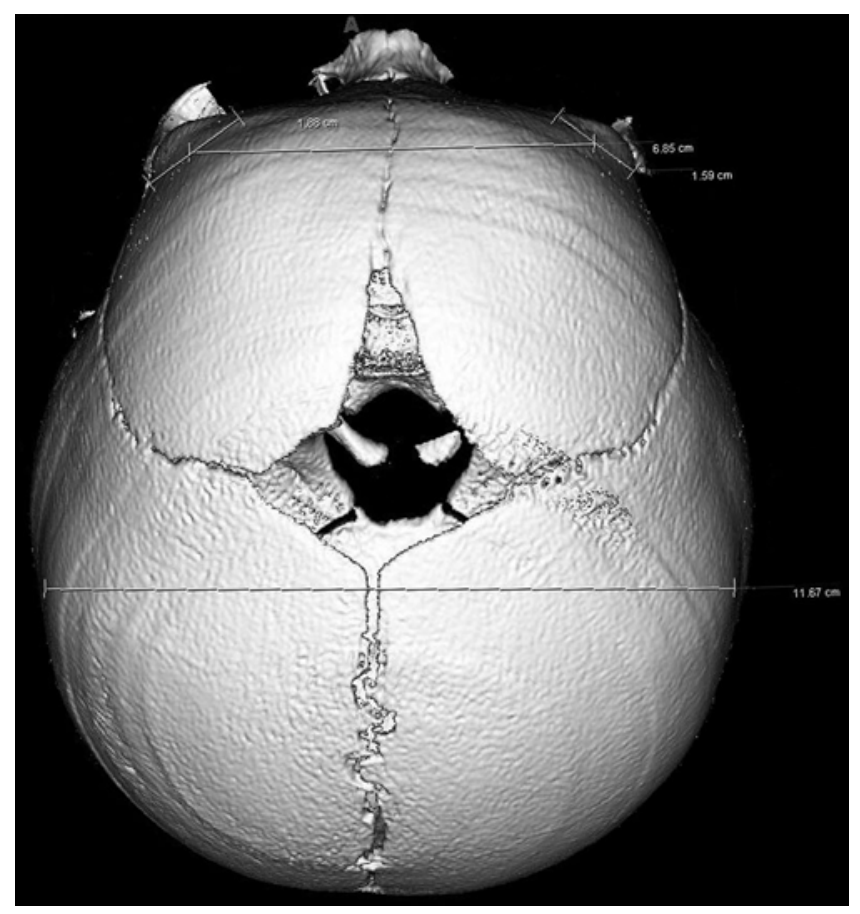

FIG. 1. Superior projection of a 3D reconstruction of a CT scan obtained in a child without craniosynostosis. Displayed are the midfrontozygomatic diameter (MFZD, $6.85 \mathrm{~cm}$ ) and the eurion-eurion diameter (EuD, 11.67 $\mathrm{cm})$. The metopic index (MI) is therefore 0.59 , which is within the range of normal based upon measurements obtained in control subjects. 
as well as to compare clinically obtained and CT-obtained measurements, MIs were compared using the paired Student t-test. Statistical significance was defined as $\mathrm{p}<0.05$. All statistical analyses were performed using Stata/IC 12 (StataCorp LP).

\section{Results}

The mean age of the 15 patients with metopic synostosis was 10.8 months (SD 3.9 months). The mean age of the 316 controls at the time the 324 scans were obtained was $11.5 \pm 7.3$ months. Of the 324 scans obtained in controls, $169(52.2 \%)$ were obtained in male patients; in comparison, $13(81.3 \%)$ of the 15 scans used for analysis of metopic synostosis were obtained in male patients. One hundred twenty-three $(38.2 \%)$ of the scans obtained in controls were obtained in white patients, 161 (50\%) were obtained in African American patients, and 38 (11.8\%) were obtained in patients identified as being of another race. Twelve (80.0\%) of the 15 patients with metopic synostosis were white, and 2 (13.3\%) were African American (Table 1).

The mean preoperative EuD for patients with metopic synostosis was $121 \mathrm{~mm}$ (SD $8 \mathrm{~mm}$ ), which was not significantly different compared with the mean EuD for controls $(121 \mathrm{~mm}$ [SD $11 \mathrm{~mm}])$. However, the mean preoperative MFZD for patients with trigonocephaly was $58 \mathrm{~mm}$ (SD 7

TABLE 1. Summary of clinical and demographic characteristics of 316 controls and 15 patients with metopic synostosis*

\begin{tabular}{|c|c|c|}
\hline Characteristic & Controls & $\begin{array}{l}\text { Pts w/ Metopic } \\
\text { Synostosis }\end{array}$ \\
\hline Age at presentation, mos & $11.5 \pm 7.3$ & $10.8 \pm 3.9$ \\
\hline \multicolumn{3}{|l|}{ Sex } \\
\hline Male & $169(54.2)$ & $12(80.0)$ \\
\hline Female & $155(45.8)$ & $3(20.0)$ \\
\hline \multicolumn{3}{|l|}{ Race/Ethnicity } \\
\hline White & $123(38.0)$ & $12(80.0)$ \\
\hline African American & $161(49.7)$ & $2(13.3)$ \\
\hline Other & $40(12.3)$ & $1(0.7)$ \\
\hline \multicolumn{3}{|l|}{ Baseline measurements } \\
\hline EuD, mm & $121 \pm 11$ & $121 \pm 8$ \\
\hline MFZD, mm & $68 \pm 5$ & $58 \pm 7$ \\
\hline $\mathrm{Ml}$ & $0.57 \pm 0.04$ & $0.48 \pm 0.05$ \\
\hline \multicolumn{3}{|l|}{ Surgery } \\
\hline Age, mos & NA & $10.3 \pm 3.7$ \\
\hline Open procedure & NA & $16(100)$ \\
\hline \multicolumn{3}{|l|}{ Follow-up imaging } \\
\hline Time from treatment, mos & NA & $7.1 \pm 8.5$ \\
\hline EuD, mm & NA & $130 \pm 9$ \\
\hline MFZD, mm & NA & $72 \pm 7$ \\
\hline $\mathrm{Ml}$ & NA & $0.55 \pm 0.03$ \\
\hline
\end{tabular}

EuD = eurion-eurion diameter; MFZD = mid-fronto-zygomatic diameter; $\mathrm{Ml}=$ metopic index; $N A=$ not applicable; pts = patients.

* Values are given as mean \pm SD or number (\%). Two separate CT studies obtained at different time points were included for 8 of the patients in the control group. Thus the number of CT scans used for comparison is 324 and demographic data are given per scan rather than per patient. $\mathrm{mm}$ ), significantly lower than that of the control cohort (69 $\mathrm{mm}$ [SD $5 \mathrm{~mm}$ ]; $\mathrm{p}<0.001)$. The EuD and the MFZD were used to calculate the MI (Table 1, Supplemental Table 1). The mean preoperative MI for patients with trigonocephaly was 0.48 (SD 0.05), compared with 0.57 (SD 0.04) in all 324 control scans $(\mathrm{p}<0.001)$ and 0.58 (SD 0.04) in a subset of 112 scans obtained in age-matched controls ( $p$ $<0.001$ ). Comparisons were also performed after the patients were stratified into 3 age groups (6-9 months, 9-12 months, and > 12 months); in each group, the mean MI for patients with trigonocephaly was significantly lower than that for the controls ( $\mathrm{p}<0.001$, Table 2).

All 15 patients underwent open anterior craniofacial reconstruction for metopic synostosis. For the 7 patients with pre- and postoperative CT scans, the mean length of time between the surgery and the postoperative CT scan was 7.1 months (SD 8.5 months). The mean postoperative MI for these 7 patients was 0.55 (SD 0.03), significantly greater than the mean preoperative MI (0.48 [SD 0.04], $\mathrm{p}$ $=0.001)$ (Table 1). Additionally, the mean postoperative MI was comparable to the MI of the controls $(\mathrm{p}=0.30)$. In Fig. 2A and $\mathrm{C}$, preoperative photographs of a representative patient are displayed, and postoperative photographs are shown in Fig. 2B and D. The corresponding 3D reconstruction of preoperative CT and the MFZD and EuD measurements are displayed in the left panel of Fig. 3; the MI derived from these measurements is 0.45 . The $3 \mathrm{D}$ reconstruction of the $\mathrm{CT}$ scan obtained on postoperative Day 1 and corresponding measurements are shown in the right panel; the corrected $\mathrm{MI}$ is 0.54 . In the 4 patients with available clinical data, the mean MI obtained from clinical measurements ( 0.55 [SD 0.02]) was comparable to that derived from CT $(0.59$ [SD 0.03], $\mathrm{p}=0.141)($ Table 3$)$.

\section{Discussion}

While once thought to account for only $3 \%$ to $4 \%$ of craniosynostosis cases, metopic synostosis is currently estimated to account for at least $10 \%$ of cases. ${ }^{15}$ There is a growing body of literature detailing techniques for surgical correction of trigonocephaly, but there is currently no standardized grading system for the degree of cranial deformity in metopic synostosis. The majority of studies focus on aesthetic markers, pre- and postoperatively. ${ }^{1,9,11,13,18}$ Although cosmetic parameters are a critical part of the clinical evaluation in trigonocephaly, the use of aesthetic markers in combination with objective measures may allow for more optimal preoperative evaluation, operative planning, and postoperative assessment of correction and need for further therapy. Many studies have described nor-

TABLE 2. Age-matched comparison of metopic index values for controls versus patients with metopic synostosis

\begin{tabular}{ccccccccc}
\hline & \multicolumn{2}{c}{ Controls } & & \multicolumn{2}{c}{ Pts w/ Metopic } \\
\cline { 2 - 3 } \cline { 6 - 8 } Age & No. of Pts & Ml & & No. of Pts & Ml & Value \\
\hline $6-9$ mos & 19 & $0.57 \pm 0.02$ & & 5 & $0.46 \pm 0.05$ & $<0.001$ \\
\hline $9-12$ mos & 29 & $0.56 \pm 0.04$ & & 7 & $0.49 \pm 0.05$ & $<0.001$ \\
\hline$>12$ mos & 64 & $0.56 \pm 0.03$ & & 3 & $0.48 \pm 0.03$ & $<0.001$ \\
\hline
\end{tabular}

$\mathrm{MI}=$ metopic index 

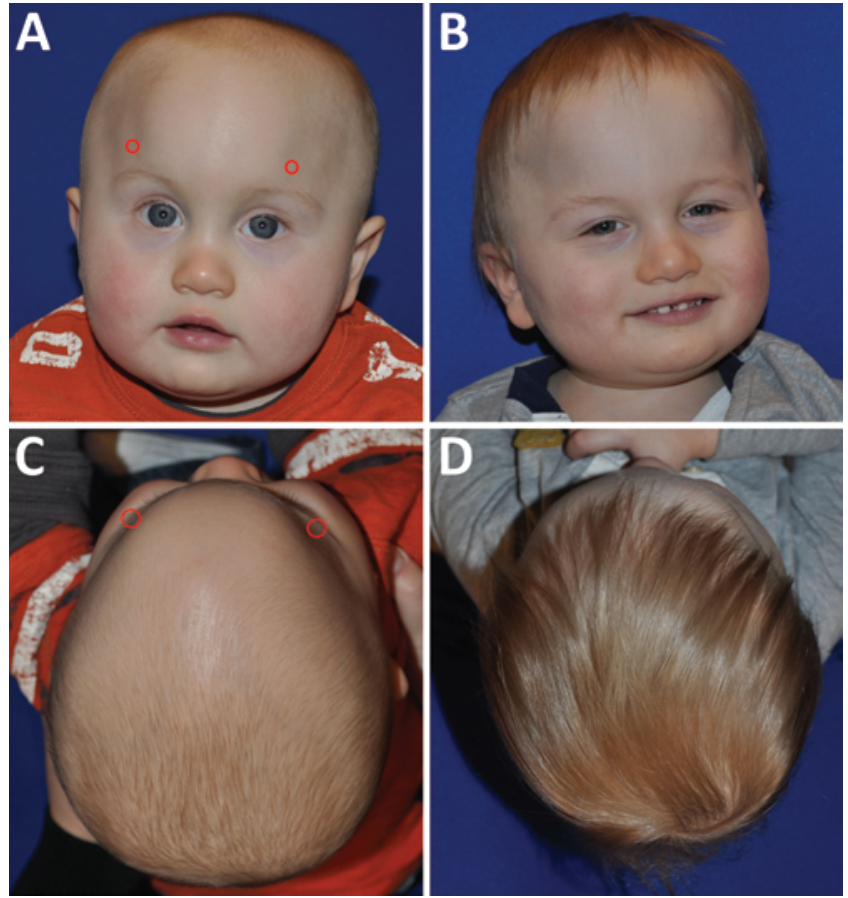

FIG. 2. Preoperative (A and C) and 1-year postoperative (B and D) views of a patient with metopic craniosynostosis. The preoperative views demonstrate classic trigonocephaly with bifrontal narrowing. In a trigonocephalic patient, the MFZD can be easily measured between the palpable recesses above the brow ridges bilaterally ( $A$ and $C$, red circles). In the clinical setting, caliper points can be placed in the recesses of the brow ridge that are visible from the superior vantage point (C). This recess is pronounced and displaced medially in infants with trigonocephaly. mative anthropometric cranial measurements and proportion indices in the pediatric population., ${ }^{3,5}$ Although 2D anthropometric measurements may not be able to fully convey the 3D cranial deformities that exist in craniosynostosis, such measurements constitute a valuable tool for the accurate and reliable assessment of craniofacial disorders. Anthropometric indices have been successfully applied to assessing cranial deformity in Down syndrome, positional plagiocephaly, and brachycephaly. ${ }^{6,21,22}$

Indeed, previous studies have demonstrated that anthropometric indices can be successfully used in the assessment of metopic synostosis. Kolar and Salter ${ }^{10}$ assessed 24 anthropometric measurements in patients with trigonocephaly and calculated 11 cranial indices. They found that the cephalic index (CI), the EuD taken over the glabella-opisthocranion diameter, did not differ significantly between controls and patients with trigonocephaly. However, they also described the frontoparietal index (FPI), calculated from the frontotemporal diameter taken over the EuD, which was significantly lower in patients with metopic synostosis. Beckett et al. ${ }^{2}$ found that the endocranial bifrontal angle varied significantly between patients with trigonocephaly and controls, and defined 2 severity grades. Wilbrand and colleagues ${ }^{20}$ described their percentile-based assessment of craniosynostosis, comparing values obtained in patients against normative values for cranial circumference, length, and width, cranial index, as well as sagittal and transversal circumference. Our goal was to describe an anthropomorphic index that would afford similar reliability and would allow for rapid calculation from both CT scans and clinical examination using values obtained with measuring calipers. Studies
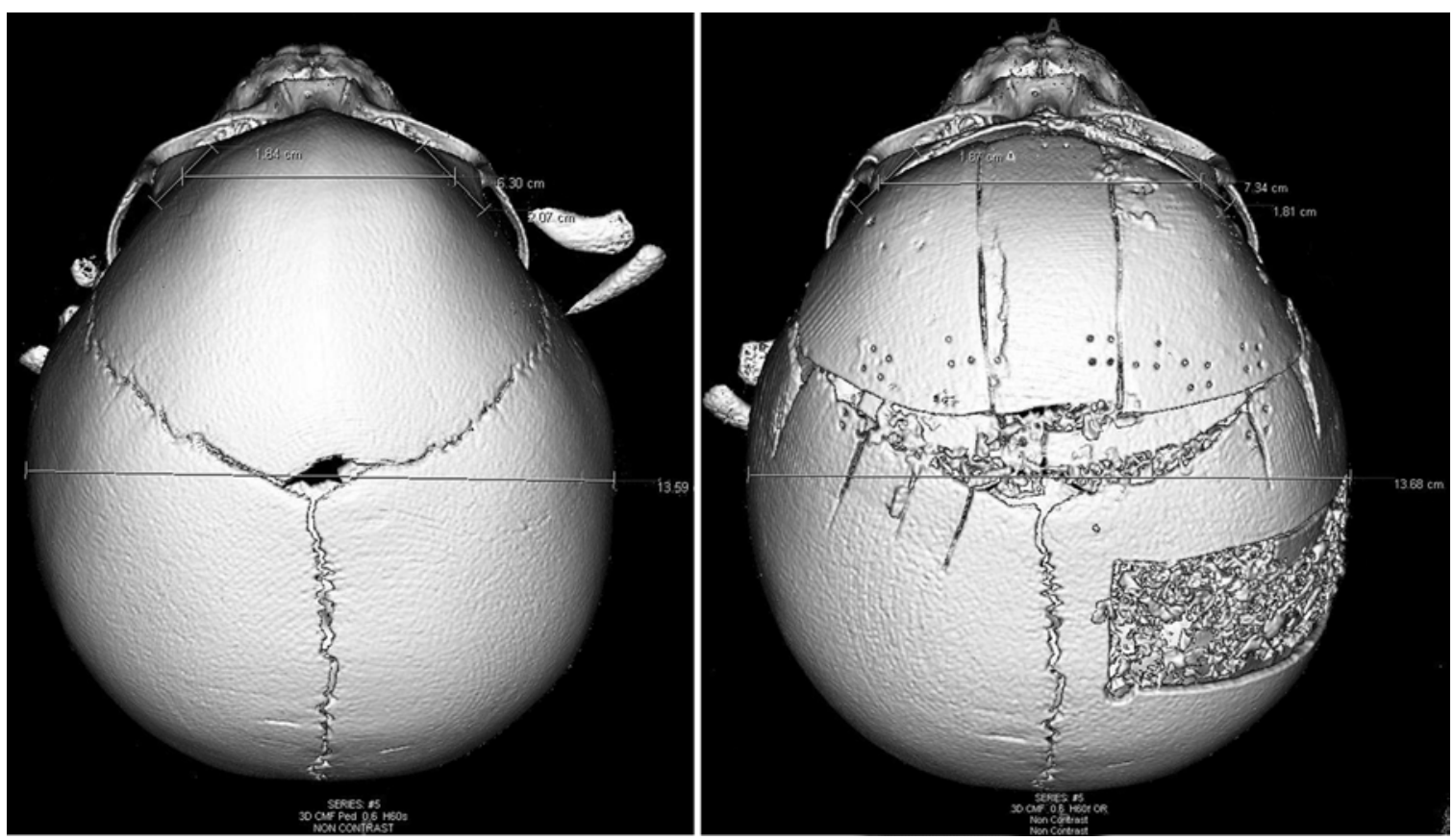

FIG. 3. Superior projections of 3D reconstructions of CT scans obtained in the patient in Fig. 2 preoperatively (left) and 1 day after surgery (right). The MI was 0.46 (MFZD $6.30 \mathrm{~cm} / E u D 13.59 \mathrm{~cm}$ ) preoperatively and significantly improved to 0.54 (MFZD $7.34 \mathrm{~cm} /$ EuD $13.68 \mathrm{~cm}$ ) postoperatively. The postoperative image demonstrates interval bifrontal contouring with rounding and widening of the MFZD. 
TABLE 3. Comparison of postoperative clinically obtained versus CT-obtained cranial measurements*

\begin{tabular}{rccccccc}
\hline \multirow{2}{*}{$\begin{array}{c}\text { Case } \\
\text { No. }\end{array}$} & \multicolumn{3}{c}{ CT-Obtained } & & \multicolumn{3}{c}{ Clinically Obtained } \\
\cline { 2 - 4 } \cline { 7 - 8 } & MFZD & EuD & MI & & MFZD & EuD & MI \\
\hline 7 & 74.5 & 137 & 0.54 & & 84 & 134 & 0.63 \\
\hline 10 & 72.5 & 130 & 0.56 & & 80 & 134 & 0.60 \\
\hline 11 & 62.0 & 116 & 0.54 & & 68 & 124 & 0.55 \\
\hline 15 & 66.6 & 118 & 0.57 & & 71 & 122 & 0.58 \\
\hline
\end{tabular}

$\mathrm{MI}=$ metopic index.

* Measurements in millimeters.

have suggested that not all cases of single-suture craniosynostosis require CT scans for evaluation. ${ }^{7}$ An anthropometric index that can be calculated from palpable cranial landmarks would not only be cost-effective but would also minimize potential side effects from ionizing radiation. In this study, we compared measurements obtained through $\mathrm{CT}$ and through physical examination in 4 patients and found no significant differences (Table 3). With the exception of 1 patient, the measurements were nearly the same with both modalities.

The metopic index (MI) was conceived by taking into account the characteristic forehead dysmorphology of patients with trigonocephaly. Compared with the FPI, we believe the MFZD more accurately captures the severity of the abnormalities of the frontoorbitozygomatic complex. In addition, the MFZ recess is readily visible and is also a more easily palpable cranial landmark, particularly in patients with trigonocephaly. While the EuD was comparable between control patients and patients with trigonocephaly, the MFZD was significantly different and accounted for the difference between the 2 groups with respect to the MI. The MI is a potentially useful tool that can be used to assess the degree of cranial deformity, but clinical judgment remains paramount in determining surgical candidates, with understood variability in measuring techniques and other patient characteristics. In particular, there remains a potential for interrater variability in determining cranial measurements in young infants by physical examination. Recently, Rinkinen and colleagues ${ }^{14}$ described a set of temporal morphomic indices (TMIs), anthropometric measurements of the temporalis muscle and temporal fat pad, that can also be derived from CT scans. Although craniosynostosis is a disorder of the cranium, the authors proposed that compensatory changes to the surrounding soft tissue are reflected in TMIs, which appear to also be specific to each subtype of craniosynostosis. ${ }^{14}$ While we believe that the MI has value in part due to its ability to be calculated from the clinical examination, in the future, assessment of cranial deformity with anthropometric cranial indices and aesthetic parameters with quantification soft tissue changes will allow for a more comprehensive evaluation of trigonocephaly severity.

\section{Study Limitations}

Although a large sample of control patients was used to define normative values for the MI, the current study is limited by the size of the metopic synostosis cohort. Furthermore, data from patients with trigonocephaly of a wider age range with multiple patients in each age group would improve generalizability. Further refinement of the MI and definition of mild, moderate, and severe subclasses of trigonocephaly will require validation in a larger cohort. Additionally, a limited number of patients received postoperative CT scans of adequate resolution to create $3 \mathrm{D}$ reconstructions, limiting the pre- and postoperative comparison of MI values. All patients in the trigonocephaly cohort underwent open surgical correction, but postoperative evaluation of patients after endoscopic repair should also be studied in the future. Despite the above limitations, this study describes a potentially useful cranial index for the assessment of metopic synostosis.

\section{Conclusions}

Because the metopic suture normally fuses during infancy, there are varying degrees of severity in head shape abnormalities associated with premature fusion. Objective and reproducible assessment of metopic synostosis is imperative to ensure appropriate patient selection for surgical repair and to aid in operative decision making and postoperative evaluation of correction and need for additional therapy. Current methods of assessing craniosynostosis are limited by their reliance on aesthetic markers, which, while important for ensuring optimal cosmesis, remain highly subjective. The metopic index (MI) is an anthropometric index that can be derived from CT scans and clinical examination of palpable cranial landmarks and is a potentially useful tool for the evaluation of trigonocephaly from metopic synostosis.

\section{References}

1. Anand A, Campion NJ, Cheshire J, Haigh T, Leckenby J, Nishikawa H, et al: Analysis of cosmetic results of metopic synostosis: concordance and interobserver variability. J Craniofac Surg 24:304-308, 2013

2. Beckett JS, Chadha P, Persing JA, Steinbacher DM: Classification of trigonocephaly in metopic synostosis. Plast Reconstr Surg 130:442e-447e, 2012

3. Christofides EA, Steinmann ME: A novel anthropometric chart for craniofacial surgery. J Craniofac Surg 21:352-357, 2010

4. Ezaldein HH, Metzler P, Persing JA, Steinbacher DM: Threedimensional orbital dysmorphology in metopic synostosis. J Plast Reconstr Aesthet Surg 67:900-905, 2014

5. Farkas LG: Accuracy of anthropometric measurements: past, present, and future. Cleft Palate Craniofac J 33:10-22, 1996

6. Farkas LG, Katic MJ, Forrest CR: Surface anatomy of the face in Down's syndrome: anthropometric proportion indices in the craniofacial regions. J Craniofac Surg 12:519-526, 2001

7. Fearon JA, Singh DJ, Beals SP, Yu JC: The diagnosis and treatment of single-sutural synostoses: are computed tomographic scans necessary? Plast Reconstr Surg 120:13271331,2007

8. Hankinson TC, Fontana EJ, Anderson RC, Feldstein NA: Surgical treatment of single-suture craniosynostosis: an argument for quantitative methods to evaluate cosmetic outcomes. J Neurosurg Pediatr 6:193-197, 2010

9. Hilling DE, Mathijssen IM, Vaandrager JM: Aesthetic results of fronto-orbital correction in trigonocephaly. J Craniofac Surg 17:1167-1174, 2006 
10. Kolar JC, Salter EM: Preoperative anthropometric dysmorphology in metopic synostosis. Am J Phys Anthropol 103:341-351, 1997

11. Kunz M, Lehner M, Heger A, Armbruster L, Weigand H, Mast G, et al: Neurodevelopmental and esthetic results in children after surgical correction of metopic suture synostosis: a single institutional experience. Childs Nerv Syst 30:1075-1082, 2014

12. Lee HQ, Hutson JM, Wray AC, Lo PA, Chong DK, Holmes $\mathrm{AD}$, et al: Changing epidemiology of nonsyndromic craniosynostosis and revisiting the risk factors. J Craniofac Surg 23:1245-1251, 2012

13. Mehta VA, Bettegowda C, Jallo GI, Ahn ES: The evolution of surgical management for craniosynostosis. Neurosurg Focus 29(6):E5, 2010

14. Rinkinen J, Wang L, Zhang P, Lisiecki J, Enchakalody B, Holcombe S, et al: Use of temporal morphomic indices as a clinically important variable in the diagnosis of nonsyndromic craniosynostosis. Ann Plast Surg 73:86-91, 2014

15. van der Meulen J: Metopic synostosis. Childs Nerv Syst 28:1359-1367, 2012

16. Vu HL, Panchal J, Parker EE, Levine NS, Francel P: The timing of physiologic closure of the metopic suture: a review of 159 patients using reconstructed 3D CT scans of the craniofacial region. J Craniofac Surg 12:527-532, 2001

17. Weinzweig J, Kirschner RE, Farley A, Reiss P, Hunter J, Whitaker LA, et al: Metopic synostosis: Defining the temporal sequence of normal suture fusion and differentiating it from synostosis on the basis of computed tomography images. Plast Reconstr Surg 112:1211-1218, 2003

18. Wes AM, Paliga JT, Goldstein JA, Whitaker LA, Bartlett SP, Taylor JA: An evaluation of complications, revisions, and long-term aesthetic outcomes in nonsyndromic metopic craniosynostosis. Plast Reconstr Surg 133:1453-1464, 2014

19. Whitaker LA, Bartlett SP, Schut L, Bruce D: Craniosynostosis: an analysis of the timing, treatment, and complications in 164 consecutive patients. Plast Reconstr Surg 80:195-212, 1987

20. Wilbrand JF, Bierther U, Nord T, Reinges M, Hahn A, Chris- tophis P, et al: Percentile-based assessment of craniosynostosis. J Craniomaxillofac Surg 42:634-640, 2014

21. Wilbrand JF, Schmidtberg K, Bierther U, Streckbein P, PonsKuehnemann J, Christophis P, et al: Clinical classification of infant nonsynostotic cranial deformity. J Pediatr 161:11201125,2012

22. Wilbrand JF, Wilbrand M, Pons-Kuehnemann J, Blecher JC, Christophis P, Howaldt HP, et al: Value and reliability of anthropometric measurements of cranial deformity in early childhood. J Craniomaxillofac Surg 39:24-29, 2011

\section{Disclosures}

The authors report no conflict of interest concerning the materials or methods used in this study or the findings specified in this paper.

\section{Author Contributions}

Conception and design: Ahn, Dorafshar. Acquisition of data: all authors. Analysis and interpretation of data: Ahn, Wang, Dorafshar. Drafting the article: Ahn, Wang. Critically revising the article: all authors. Reviewed submitted version of manuscript: all authors. Approved the final version of the manuscript on behalf of all authors: Ahn. Statistical analysis: Wang.

\section{Supplemental Information}

Online-Only Content

Supplemental material is available with the online version of the article.

Supplemental Table 1. http://thejns.org/doi/suppl/10.3171/ 2016.2.PEDS15524

\section{Correspondence}

Edward S. Ahn, Division of Pediatric Neurosurgery, Johns Hopkins University School of Medicine, 600 North Wolfe St., Phipps 560A, Baltimore, MD 21287. email: eahn4@jhmi.edu. 